\title{
A CLASS OF SOBOLEV TYPE INEQUALITIES*
}

\author{
GU-JI TIAN ${ }^{\dagger}$ AND XU-JIA WANG W $^{\ddagger}$
}

\begin{abstract}
In this paper, we prove that for any strictly convex polynomial, or more generally any strictly convex function satisfying appropriate conditions, there is an associated Sobolev type inequality.
\end{abstract}

Key words. Sobolev inequality, Green function, elliptic equation.

AMS subject classifications. 46E $35,45 \mathrm{~J} 20,35 \mathrm{~J} 60$

1. Introduction. Sobolev type inequalities play a crucial role in the study of local behavior of solutions to elliptic partial differential equations. To prove the local estimates such as the Harnack inequality and Hölder continuity, usually one first proves related Sobolev type inequalities [GT]. In particular various weighted Sobolev type inequalities have been established in the study of degenerate elliptic equations, such as those generated by vector fields which satisfy Hormander's condition [CDG, FGW1, FGW2, FL, GN, L1].

In this paper we consider the inverse problem. We prove that the Sobolev inequality can also be obtained from the Harnack inequality. We will prove a family of Sobolev type inequalities from the Harnack inequality for the linearized Monge-Ampere equation, established by Caffarelli and Gutierrez [CG2]. We prove that for each convex function satisfying appropriate conditions, there is an associate Sobolev type inequality. For example the classical Sobolev embedding $W_{0}^{1,2}(\Omega) \hookrightarrow L^{2 n /(n-2)}(\Omega)$ is associated with the quadratic function $\varphi(x)=\frac{1}{2}|x|^{2}$. Here we state the results for convex polynomials.

TheOREm 1.1. Let $\varphi$ be a strictly convex polynomial in $\mathbb{R}^{n}, n \geq 3$. Denote by $A=\left\{a_{i j}\right\}$ the cofactor matrix of $D^{2} \varphi$. Then for any bounded domain $\Omega \subset B_{R}(0)$ and any function $u \in C_{0}^{\infty}(\Omega)$,

$$
\left[\int_{\Omega}|u|^{p} \nu d x\right]^{\frac{1}{p}} \leq C\left[\int_{\Omega} \sum_{i, j} a_{i j} u_{x_{i}} u_{x_{j}} d x\right]^{\frac{1}{2}},
$$

where

$$
\nu=\operatorname{det} D^{2} \varphi
$$

and the constant $p>2$ depends only on $n$ and $\varphi$, and $C$ also depends on $R$.

Theorem 1.1 is a special case of Theorem 3.1 below. In Theorem 3.1 we show that (1.1) holds for any strictly convex function $\varphi$ provided $\nu$ satisfies (3.14) and a structure condition $\mathbb{C} \mathbb{G}$ in Section 3. Let us consider some examples.

* Received April 23, 2008; accepted for publication June 5, 2008. This work was supported by NSFC Grant 10571069 and ARC DP0664517 and DP0879422.

$\dagger$ Wuhan Institute of Physics and Mathematics, Chinese Academy of Sciences, Wuhan 430071, China (tianguji@wipm.ac.cn).

$\ddagger$ Centre for Mathematics and Its Applications, The Australian National University, Canberra, ACT 0200, Australia (X.J.Wang@maths.anu.edu.au). 
EXAMPLE 1. Let

$$
\varphi(x)=\left|x_{1}\right|^{\alpha+2}+\sum_{i>1} x_{i}^{2},
$$

where $\alpha>-1$. Then for any $u \in C_{0}^{\infty}(\Omega)$,

$$
\left[\int_{\Omega}|u|^{p}\left|x_{1}\right|^{\alpha} d x\right]^{\frac{1}{p}} \leq C\left[\int_{\Omega} u_{x_{1}}^{2}+\left|x_{1}\right|^{\alpha} \sum_{i>1} u_{x_{i}}^{2} d x\right]^{\frac{1}{2}} .
$$

The power $p$ can easily computed by (3.33) and (3.40). The left hand side of (1.4) can be replaced by the norm $\|u\|_{L^{p}(\Omega)}$ for a different $p>2$ given by (3.37), see Remark 3.4. We remark that the functional on the right hand side of (1.4) is related to the Grushin type operator [FGW2, M].

Example 2. More generally, let

$$
\varphi(x)=\left|x_{1}\right|^{\alpha_{1}+2}+\cdots+\left|x_{n}\right|^{\alpha_{n}+2},
$$

where $\alpha_{i}>-1$ satisfy (3.42). Then we obtain

$$
\left[\int_{\Omega}|u|^{p} \sigma d x\right]^{\frac{1}{p}} \leq C\left[\int_{\Omega} \sum_{i} \sigma_{i} u_{x_{i}}^{2} d x\right]^{\frac{1}{2}},
$$

where $\sigma(x)=\prod\left|x_{i}\right|^{\alpha_{i}}, \sigma_{i}(x)=\sigma(x) / x_{i}^{\alpha_{i}}$. When $\alpha_{1}, \cdots, \alpha_{n}$ satisfies the condition (3.41), we can drop the weight $\sigma$ and (1.6) holds for a different $p>2$, see Remark 3.4. Note also that (1.6) holds obviously if one replaces $\sigma_{i}$ by another function $\hat{\sigma}_{i}$ which satisfies $\hat{\sigma}_{i}(x) \geq 0, \not \equiv 0$ and $\hat{\sigma}_{i} \geq \sigma_{i}$, where $i=1,2, \cdots, n$. Hence (1.6) represents a family of Sobolev type inequalities.

Example 3. Let $\varphi$ be a strictly convex function satisfying

$$
C_{1} \leq \operatorname{det} D^{2} \varphi \leq C_{2} \text { in } \Omega
$$

for two positive constants $C_{1}, C_{2}$, then for any $u \in C_{0}^{\infty}(\Omega)$,

$$
\left[\int_{\Omega}|u|^{\frac{2 n}{n-2}} d x\right]^{\frac{n-2}{2 n}} \leq C\left[\int_{\Omega} a_{i j}(x) u_{x_{i}} u_{x_{j}} d x\right]^{\frac{1}{2}}
$$

provided $a_{i j}$ are integrable functions. Obviously the exponent in (1.8) is sharp. Note that even in dimension two, there are many nonsmooth, strictly convex functions satisfying (1.7). For example, the following two functions satisfy (1.7) in $B_{r}(0)$ for some $r>0[\mathrm{~W}]$,

$$
\varphi(x)=\frac{x_{1}^{2}}{\left.\log |\log | x\right|^{2} \mid}+\left.x_{2}^{2} \log |\log | x\right|^{2} \mid
$$

and

$$
\varphi(x)= \begin{cases}x_{1}^{4}+\frac{3}{2} x_{2}^{2} / x_{1}^{2} & \text { if }\left|x_{2}\right|<\left|x_{1}\right|^{3} \\ \frac{1}{2} x_{1}^{2} x_{2}^{2 / 3}+2 x_{2}^{4 / 3} & \text { if }\left|x_{2}\right|>\left|x_{1}\right|^{3} .\end{cases}
$$

Both functions $\varphi$ are $C^{1, \alpha}$ but not $C^{1,1}$ smooth, and the corresponding matrix $\left\{a_{i j}\right\}$ is both degenerate and singular.

We would like to point out that for most convex functions $\varphi$, the associated Sobolev type inequalities we obtained are either new or improve previous ones. Our proof is based on a crucial lemma (Lemma 2.1), of which the proof is essentially due to Carron $[\mathrm{C}]$ but we made some necessary modifications. Lemma 2.1 shows 
that the Sobolev inequality follows from a decay estimate (2.2) of the corresponding Green function. This argument also applies to more general elliptic operators, namely we can also obtain the Sobolev type inequality from the Harnack inequality for other degenerate or singular linear elliptic equations. In particular our argument also applies to linear elliptic equations on manifolds. Note also that in Lemma 2.1 we allow a weight $\mu$, which can be the function $\nu$ given in (1.2) as in Theorems 1.1 and 3.1 , or $\nu \equiv 1$ as in Theorem 3.2. But other choices are also possible.

This paper is arranged as follows. In Section 2 we prove the crucial lemma. In Section 3 we use the Harnack inequality to establish a decay estimate for the Green function.

This paper is dedicated to Professor Neil Trudinger on the occasion of his 65th birthday. We would like to express our gratitude for his enthusiasm, influence, and leadership in elliptic partial differential equations. In particular the second author would like to thank him for his friendship in their collaboration over the last ten years.

2. A crucial lemma. Let $L=\sum \partial_{x_{i}}\left(a_{i j}(x) \partial_{x_{j}}\right)$ be a linear elliptic operator in a bounded domain $\Omega$. We assume that $L$ is uniformly elliptic but our argument below does not depend on the upper and lower bounds of the eigenvalues of the matrix $\left\{a_{i j}\right\}$. Let $G(x, y)$ be the Green function of $L$ in $\Omega$, namely $G(\cdot, y)$ is a positive solution of

$$
\begin{aligned}
-L[G(\cdot, y)] & =\delta_{y} \quad \text { in } \quad \Omega, \\
G(\cdot, y) & =0 \text { on } \partial \Omega
\end{aligned}
$$

where $\delta_{y}$ is the Dirac measure at $y \in \Omega$. For an integrable function $\mu$, we also denote by $\mu$ the corresponding measure, that is for any Borel set $S \subset \mathbb{R}^{n}$,

$$
\mu(S)=\int_{S} d \mu=\int_{S} \mu d x .
$$

LEMMA 2.1. Suppose there is an integrable, almost everywhere positive function $\mu$ such that for any $t>0$,

$$
\mu\{x \in \Omega: G(x, y)>t\} \leq K t^{-p / 2},
$$

where $p>2$ and $K>0$ are constants. Suppose also that $\mu$ satisfies the doubling condition, namely there exists a constant $b>0$ such that

$$
\mu[B(x, r)] \geq b \mu[B(x, 2 r)]
$$

for any ball $B(x, 2 r) \Subset \Omega$. Then for any smooth function $u \in C_{0}^{\infty}(\Omega)$, we have the inequality

$$
\left[\int_{\Omega}|u|^{p} d \mu\right]^{\frac{1}{p}} \leq C\left[\int_{\Omega} \sum a_{i j} u_{x_{i}} u_{x_{j}} d x\right]^{\frac{1}{2}}
$$

where the constant $C$ depends only on $n, p, b$, and $K$.

Proof. Our proof is inspired by that in [C], see also Chapter 8 of [H]. For any open set $U \subset \Omega$, let $\psi_{1}=\psi_{1, U}$ be the first eigenfunction, and $\lambda_{1}=\lambda_{1, U}$ the first 
eigenvalue, of the operator $L$ in $U$, namely

$$
\begin{aligned}
L\left[\psi_{1}\right] & =\lambda_{1} \mu \psi_{1} \text { in } U, \\
\psi_{1} & =0 \text { on } \partial U .
\end{aligned}
$$

Let $G_{U}$ be the Green function of $L$ in $U$. Then we have

$$
\psi_{1}(y)=\lambda_{1} \int_{U} \mu G_{U}(x, y) \psi_{1}(x) d x .
$$

Suppose $\psi_{1}$ attains its maximum at $\bar{y}$. Letting $y=\bar{y}$ in the above formula, we obtain

$$
1 \leq \lambda_{1} \int_{U} \mu G_{U}(x, y) d x
$$

By $(2.2)$,

$$
\begin{aligned}
\int_{U} \mu G_{U}(x, y) d x & =\int_{0}^{\infty} \mu\left\{G_{U}(\cdot, y)>t\right\} \\
& \leq \int_{0}^{\infty} \min \left\{\mu(U), K t^{-p / 2}\right\} \\
& =T \mu(U)+K \int_{T}^{\infty} t^{-p / 2} \\
& \leq C[\mu(U)]^{1-\frac{2}{p}}
\end{aligned}
$$

where $T$ is chosen such that $K T^{-p / 2}=\mu(U)$. It follows that

$$
\lambda_{1, U}[\mu(U)]^{1-\frac{2}{p}} \geq C^{-1} .
$$

Denote

$$
\begin{aligned}
& c^{*}=\inf _{U \Subset \Omega} \lambda_{1, U}[\mu(U)]^{1-\frac{2}{p}}, \\
& s^{*}=\inf \left\{\int_{\Omega} a_{i j} u_{i} u_{j}: u \in C_{0}^{1}(\bar{\Omega}), \int_{\Omega} F(u) d \mu=1\right\},
\end{aligned}
$$

where $F(u)=\int_{0}^{u} f(t) d t$ and

$$
f(t)= \begin{cases}|t|^{p-1} & \text { if }|t|<k \\ k^{p-1} & \text { if }|t| \geq k\end{cases}
$$

and $k>1$ is a constant which will be sent to infinity. Suppose the infimum (2.8) is attained by a positive function $v=v_{k} \in C^{1}(\bar{\Omega})$. Then $v$ vanishes on $\partial \Omega$ and satisfies the equation

$$
-L[v]=\hat{\lambda} \mu f(v) \text { in } \Omega,
$$

where $\hat{\lambda}$ is the Langrange multiplier. By our choice of $f, \frac{1}{p} f(v) v \leq F(v) \leq f(v) v$. Hence multiplying (2.9) by $v$ and taking integration we obtain

$$
\hat{\lambda} \leq s^{*} \leq p \hat{\lambda} .
$$

We point out here that we only use the existence of a Hölder continuous solution $v$ but do not need any a priori bound for this solution. Hence we do not need any a priori bounds for the eigenvalues of $\left\{a_{i j}\right\}$. 
Denote $M=\|v\|_{L^{\infty}}, \Omega_{t}=\{v(x)>M-t\}$. For $t \in(0, M)$, we claim

$$
\mu\left(\Omega_{t}\right) \geq\left(\frac{t c^{*}}{2 s^{*} M^{p-1}}\right)^{\frac{p}{p-2}} .
$$

Indeed, by (2.9),

$$
\begin{aligned}
\lambda_{1}\left(\Omega_{t}\right) & \leq \frac{\int_{\Omega_{t}} a_{i j} v_{i} v_{j} d x}{\int_{\Omega_{t}}(v-M+t)^{2} d \mu} \\
& =\frac{\int_{\Omega_{t}}(v-M+t)(-L v) d x}{\int_{\Omega_{t}}(v-M+t)^{2} d \mu} \\
& \leq s^{*} M^{p-1} \frac{\int_{\Omega_{t}}(v-M+t) d \mu}{\int_{\Omega_{t}}(v-M+t)^{2} d \mu} \\
& \leq s^{*} M^{p-1}\left(\frac{\mu\left(\Omega_{t}\right)}{\int_{\Omega_{t}}(v-M+t)^{2} d \mu}\right)^{1 / 2} .
\end{aligned}
$$

Noting that

$$
\int_{\Omega_{t}}(v-M+t)^{2} d \mu \geq\left(\frac{t}{2}\right)^{2} \mu\left(\Omega_{t / 2}\right)
$$

we obtain from (2.7),

$$
c^{*} \mu\left(\Omega_{t}\right)^{\frac{2}{p}-1} \leq \lambda_{1}\left(\Omega_{t}\right) \leq \frac{2 s^{*} M^{p-1}}{t}\left(\frac{\mu\left(\Omega_{t}\right)}{\mu\left(\Omega_{t / 2}\right)}\right)^{1 / 2} .
$$

It follows that

$$
\mu\left(\Omega_{t}\right) \geq \beta \mu\left(\Omega_{t / 2}\right)^{\frac{p}{3 p-4}},
$$

where

$$
\beta=\left(\frac{t c^{*}}{2 s^{*} M^{p-1}}\right)^{\frac{2 p}{3 p-4}}
$$

By iteration,

$$
\mu\left(\Omega_{t}\right) \geq \beta^{\sum_{k=0}^{m-1}\left(\frac{p}{3 p-4}\right)^{k}} \mu\left(\Omega_{t / 2^{m}}\right)^{\left(\frac{p}{3 p-4}\right)^{m}} .
$$

Let $a=\sup _{\Omega}|D v|$. We have $\Omega_{t / 2^{m}} \supset B\left(x_{0}, \frac{t}{2^{m} a}\right)$, where $x_{0}$ is the maximum point of $v$. Hence by the doubling condition (2.3),

$$
\mu\left[\Omega_{t / 2^{m}}\right] \geq \mu\left[B\left(x_{0}, \frac{t}{2^{m} a}\right)\right] \geq b^{m-m_{0}} \mu\left[B\left(x_{0}, \frac{t}{2^{m_{0}} a}\right)\right],
$$

where $m_{0}$ is the smallest integer such that $B\left(x_{0}, \frac{t}{2^{m_{0} a}}\right) \subset \Omega$. Recalling that $p>2$, we obtain

$$
\mu\left(\Omega_{t / 2^{m}}\right)^{\left(\frac{p}{3 p-4}\right)^{m}} \rightarrow 1
$$

as $m \rightarrow \infty$. Therefore we get

$$
\mu\left(\Omega_{t}\right) \geq \beta^{\sum_{k=0}^{\infty}\left(\frac{p}{3 p-4}\right)^{k}}=\beta^{\frac{3 p-4}{2(p-2)}}=\left(\frac{t c^{*}}{2 s^{*} M^{p-1}}\right)^{\frac{p}{p-2}}
$$

and so (2.11) is proved. 
Now we have

$$
\begin{aligned}
1=\int_{\Omega} F(v) d \mu & =\int_{0}^{F(M)} \mu\{x \in \Omega: F(v)(x)>t\} d t \\
& =\int_{0}^{M} \mu\{x \in \Omega: v(x)>t\} F^{\prime}(t) d t \\
& =\int_{0}^{M} \mu\{x \in \Omega: v(x)>M-t\} F^{\prime}(M-t) d t \\
& =\int_{0}^{M} \mu\left(\Omega_{t}\right) F^{\prime}(M-t) d t \\
& \geq\left(\frac{c^{*}}{2 s^{*} M^{p-1}}\right)^{\frac{p}{p-2}} \int_{0}^{M} t^{\frac{p}{p-2}} F^{\prime}(M-t) d t \\
& =\left(\frac{c^{*}}{2 s^{*}}\right)^{\frac{p}{p-2}} \int_{0}^{1} t^{\frac{p}{p-2}} \frac{F^{\prime}(M(1-t))}{M^{p-1}} d t .
\end{aligned}
$$

Denote $\eta=k / M$. By our definition of $f$, we have

$$
F^{\prime}(M(1-t))= \begin{cases}p M^{p-1}(1-t)^{p-1} & \text { if } 1-\eta<t<1 \\ k^{p-1} & \text { if } 0<t<1-\eta .\end{cases}
$$

Hence we have

$$
\int_{0}^{1} t^{\frac{p}{p-2}} \frac{F^{\prime}(M(1-t))}{M^{p-1}} d t=\int_{0}^{1-\eta} t^{\frac{p}{p-2}} \eta^{p-1}+p \int_{1-\eta}^{1} t^{\frac{p}{p-2}}(1-t)^{p-1} .
$$

Recalling that $\int_{\Omega} F(u) d \mu=1$, we have

$$
1=\int_{\Omega} F(v) d \mu \geq \frac{1}{p} \int_{\omega} k^{p} d \mu=\frac{1}{p} k^{p} \mu(\omega),
$$

namely

$$
\mu(\omega) \leq p k^{-p},
$$

where $\omega=\{v>k\}$. Recalling also that $v$ satisfies the equation

$$
L(v)=\hat{\lambda} k^{p-1} \mu \quad \text { in } \omega
$$

and by (2.10), $\hat{\lambda} \leq s^{*} \leq C$, hence by the boundary condition $v=k$ on $\partial \omega$ we have

$$
\begin{aligned}
v(x) & \leq k+\hat{\lambda} k^{p-1} \int_{\omega} G(x, y) d \mu \\
& \leq k+C k^{p-1} \mu(\omega)^{1-\frac{2}{p}},
\end{aligned}
$$

where $G$ is the Green function in $\omega$ and we have used the estimate (2.5). By (2.14), it follows that

$$
M=\sup v \leq(1+C) k .
$$

Therefore we obtain $\eta=\frac{k}{M} \geq(1+C)^{-1}$. By (2.13) we therefore obtain

$$
\int_{0}^{1} t^{\frac{p}{p-2}} \frac{F^{\prime}(M(1-t))}{M^{p-1}} d t \geq C>0
$$


Consequently we obtain

$$
s^{*} \geq C_{1} c^{*}
$$

From (2.8) we obtain

$$
\int_{\Omega} a_{i j} u_{i} u_{j} d x \geq C_{1} c^{*}\left[\int_{\Omega} f(u)|u| d \mu\right]^{2 / p}
$$

for any $u \in C_{0}^{1}(\Omega)$. Sending $k \rightarrow \infty$, we obtain (2.4).

REMARK 2.1. In (2.8) we may choose $F(u)=|u|^{p-\varepsilon}$ and letting $\varepsilon \rightarrow 0[\mathrm{H}]$. Indeed, since $p \leq \frac{2 n}{n-2}$, by the Sobolev embedding $W^{1,2}(\Omega) \hookrightarrow L^{\frac{2 n}{n-2}}(\Omega)$, the infimum (2.8) is attained by a minimizer $v_{\varepsilon}$. By the above argument we then obtain

$$
\left[\int_{\Omega}|u|^{p-\varepsilon} d \mu\right]^{\frac{2}{p-\varepsilon}} \leq C \int_{\Omega} a_{i j} u_{i} u_{j} d x,
$$

where $C$ is a constant independent of $\varepsilon$. Sending $\varepsilon \rightarrow 0$ we also obtain (2.4). Our modification in (2.8) is to avoid the use of the Sobolev embedding $W^{1,2}(\Omega) \hookrightarrow L^{\frac{2 n}{n-2}}(\Omega)$.

3. Estimate for the Green function. The purpose of this section is to verify the decay estimate (2.2) for the Green function of the linearized Monge-Ampere equation. By the Harnack inequality of Caffarelli and Gutierrez [CG2], we can prove a more general Wolff potential estimate for functions which is sub-harmonic with respect to the linear elliptic operator $L_{\varphi}$, as in [TW1] for quasilinear subelliptic equations. For the linearized Monge-Ampere equation, it suffices to replace the metric ball in [TW1] by sub-level sets of the convex function $\varphi$. We refer the reader to [KM, L, TW1] for the Wolff potential estimate and [CW, FS, FJK, L2] for estimates for Green functions.

Let $\varphi$ be a smooth, strictly convex function. The linearized Monge-Ampere equation relative to the function $\varphi$ is given by

$$
L_{\varphi}[u]=f(x),
$$

where

$$
\begin{aligned}
L_{\varphi}[u] & =\sum_{i, j} \partial_{x_{i}}\left(a_{i j}(x) \partial_{x_{j}} u\right) \\
& =\sum_{i, j} a_{i j}(x) \partial_{x_{i}} \partial_{x_{j}} u,
\end{aligned}
$$

and $\left\{a_{i j}\right\}$ is the cofactor matrix of $D^{2} \varphi$. The second equality is due to that $\left\{a_{i j}\right\}$ is of divergence free, i.e.,

$$
\sum_{i} \partial_{x_{i}} a_{i j}(x)=0 \quad \forall j
$$

Denote

$$
\nu=\operatorname{det} D^{2} \varphi
$$

Assume that $\nu$ satisfies the structure condition:

$\mathbb{C} \mathbb{G}$ For any given $\varepsilon>0$, there exists $\delta>0$ such that for any convex set $S \subset \Omega$ and any set $E \subset S$, if $|E| \leq \delta|S|$, then

$$
\int_{E} \nu d x \leq \varepsilon \int_{S} \nu d x
$$

where $|\cdot|$ denotes the Lebesgue measure. 
When $\varphi$ satisfies the condition $\mathbb{C} \mathbb{G}$, Caffarelli and Gutierrez [CG2] proved a Harnack inequality for positive solutions to the equation

$$
L_{\varphi}[u]=0 .
$$

Their Harnack inequality can be stated as follows.

Lemma 3.1. Let $\varphi$ be a smooth, strictly convex function in $\Omega$ satisfying condition $\mathbb{C} \mathbb{G}$. Let $u$ be a positive solution to (3.3) in the sub-level set

$$
S_{\varphi}(y, h):=\left\{x \in \Omega: \varphi(x)<\ell_{y}(x)+h\right\},
$$

where $\ell_{y}$ is the tangent plane of $\varphi$ at $y$. If $S_{\varphi}(y, h)$ is strictly contained in $\Omega$, we have

$$
\sup _{S_{\varphi}(y, h / 2)} u \leq C \inf _{S_{\varphi}(y, h / 2)} u,
$$

where $C$ is a constant depending only on $n$ and the structure condition $\mathbb{C} \mathbb{G}$.

It is convenient to use the above Harnack inequality in a normalized form. Let $U$ be a bounded convex domain. There is a unique ellipsoid $E$, called the minimum ellipsoid of $U$, which attains the minimum volume among all ellipsoids containing $U$. Moreover

$$
\frac{1}{n} E \subset U \subset E,
$$

where $\alpha E$ denotes the $\alpha$-dilation of $E$ with respect to its center. Choosing the coordinates properly, we assume that $E$ is given by

$$
E=\left\{\sum \frac{x_{i}^{2}}{r_{i}^{2}}<1\right\}
$$

Making the linear transform $x \rightarrow \hat{x}=T x$,

$$
\hat{x}_{i}=x_{i} / r_{i}, \quad i=1,2, \cdots, n,
$$

such that $T(E)$ is the unit ball. Also let

$$
\hat{\varphi}(\hat{x})=\frac{1}{h}\left[\varphi(x)-\ell_{y}-h\right]
$$

such that

$$
\hat{\varphi}_{\left.\right|_{\partial \hat{U}}}=0, \quad \inf _{\hat{U}} \hat{\varphi}=-1,
$$

where $\hat{U}=T(U)$. In the following we say $\hat{\varphi}$ and $\hat{U}$ are normalized if the minimum ellipsoid of $\hat{U}$ is the unit ball and $\hat{\varphi}$ satisfies (3.10).

Note that the structure condition $\mathbb{C} \mathbb{G}$ is invariant under the above changes. Under condition $\mathbb{C} \mathbb{G}$, the function $\hat{\varphi}$ is strictly convex and uniformly Hölder continuous in $\hat{U}$ with Hölder exponent $1 / n[\mathrm{P}]$. Therefore by the finite covering [CG1] and the invariance of the Harnack inequality under linear transforms, we see that if $u$ is a positive solution to $(3.3)$ in $S_{\varphi}(y, h)-S_{\varphi}(y, h / 2)$, then

$$
\sup _{\partial S_{\varphi}(y, 3 h / 4)} u \leq C \inf _{\partial S_{\varphi}(y, 3 h / 4)} u .
$$

The next lemma is related to capacity estimate in potential theory. 
LEMma 3.2. Let $\hat{\varphi}$ be a normalized function defined in a normalized domain $\Omega$. Suppose $\hat{\varphi}$ satisfies the condition $\mathbb{C} \mathbb{G}$. Let $w$ be the solution of

$$
\begin{aligned}
L_{\hat{\varphi}}[w] & =0 \quad \text { in } \quad U, \\
w & =\hat{\varphi} \quad \text { on } \quad \partial U,
\end{aligned}
$$

where $U=\left\{x \in \Omega:-\frac{1}{2}<\hat{\varphi}(x)<0\right\}$. Then

$$
C_{1} \leq \int_{\partial \Omega} \sum_{i, j} a_{i j}(x) w_{i} \gamma_{j} \leq C_{2}
$$

where $\gamma$ is the unit outer normal of $\partial \Omega$.

Proof. The condition $\mathbb{C} \mathbb{G}$ implies that $\nu=\operatorname{det} D^{2} \hat{\varphi}$ satisfies the doubling condition, namely $\nu(\Omega) \leq C \nu\left(\Omega_{1 / 2}\right)$, where $\Omega_{t}=\{x \in \Omega: \hat{\varphi}(x)<-t\}, t \in(0,1)$. Since $\hat{\varphi}$ and $\Omega$ are normalized, we have $\operatorname{dist}\left(\Omega_{1 / 2}, \partial \Omega\right)>C$ and $\nu\left(\Omega_{1 / 2}\right) \leq C$ for a different constant $C$.

Observing that $L_{\hat{\varphi}}[\hat{\varphi}]=n \operatorname{det} D^{2} \hat{\varphi} \geq 0$ and $w=\hat{\varphi}=0$ on $\partial U$, by the comparison principle we have $0 \geq w \geq \hat{\varphi}$ in $U$. Hence

$$
\begin{aligned}
\int_{\partial \Omega} \sum_{i, j} a_{i j}(x) w_{i} \gamma_{j} & \leq \int_{\partial \Omega} \sum_{i, j} a_{i j}(x) \hat{\varphi}_{i} \gamma_{j} \\
& =n \int_{\Omega} \operatorname{det} D^{2} \hat{\varphi} \leq C .
\end{aligned}
$$

We obtain the second inequality. To prove the first inequality, we extend $w$ smoothly to the whole domain $\Omega$. Noting that $L_{\hat{\varphi}}[w]=0$ in $U$, by (3.2) we have

$$
\begin{aligned}
\int_{\partial \Omega} \sum_{i, j} a_{i j}(x) w_{i} \gamma_{j} & =\int_{\partial \Omega_{1 / 2}} \sum_{i, j} a_{i j}(x) w_{i} \gamma_{j} \\
& \geq \int_{\partial \Omega_{1 / 2}} \sum_{i, j} a_{i j}(x) \hat{\varphi}_{i} \gamma_{j} \\
& =n \int_{\Omega_{1 / 2}} \operatorname{det} D^{2} \hat{\varphi} \geq C
\end{aligned}
$$

We are ready to prove (2.2) for the linearized Monge-Ampere equation.

LEMMA 3.3. Let $\varphi$ be a smooth, strictly convex function defined in a neighborhood of $\bar{\Omega}$. Suppose $\varphi$ satisfies condition $\mathbb{C} \mathbb{G}$. Let $G(\cdot, y)$ be the Green function of $L_{\varphi}$ in $\Omega$, where $y \in \Omega$. Suppose for any sub-level set $S_{\varphi}(y, h) \subset \subset \Omega$,

$$
C_{1}\left|S_{h}\right|^{1+\theta} \leq \nu\left(S_{h}\right) \leq C_{2}\left|S_{h}\right|^{\frac{1}{n-1}+\sigma}, \quad S_{h}:=S_{\varphi}(y, h),
$$

where $\theta \geq 0, C_{1}, C_{2}, \sigma>0$ are constants. Then we have

$$
\nu\{x \in \Omega: G(x, y)>t\} \leq K t^{-\frac{n(1+\theta)}{(n-1)(1+\theta)-1}} .
$$

Proof. Since $\varphi$ is defined in a neighborhood of $\bar{\Omega}$, by considering the Green function of $L_{\varphi}$ in a larger domain we may assume that $\operatorname{dist}(y, \partial \Omega) \geq c_{0}$.

By the Harnack inequality (3.11), we have

$$
\sup _{\partial S_{h}} G(\cdot, y) \leq C \inf _{\partial S_{h}} G(\cdot, y)
$$


provided $S_{2 h}$ is compactly contained in $\Omega$. For any large $t>1$, let $h$ such that

$$
\inf _{\partial S_{h}} G(\cdot, y)=t .
$$

Let $E$ be the minimum ellipsoid of $S_{2 h}$. Suppose $E$ is given by (3.7). Let $\hat{x}=T x$ be the linear transform given in (3.8), and let

$$
\begin{aligned}
& \hat{\varphi}=\left[\varphi-\ell_{y}-2 h\right] / r\left[\nu\left(S_{2 h}\right)\right]^{1 / n}, \\
& \hat{u}=\left[u-\inf _{S_{2 h}} u\right] / r\left[\nu\left(S_{2 h}\right)\right]^{1 / n},
\end{aligned}
$$

where $r=\left(r_{1} \cdots r_{n}\right)^{1 / n}$. Then equations (1.2) and (3.1) change to

$$
\begin{aligned}
& \operatorname{det} D^{2} \hat{\varphi}=\hat{\nu}, \\
& L_{\hat{\varphi}}[\hat{u}]=\hat{f},
\end{aligned}
$$

where

$$
\hat{\nu}(\hat{x})=\frac{r^{n}}{\nu\left(S_{2 h}\right)} \nu(x), \quad \hat{f}(\hat{x})=\frac{r^{n}}{\nu\left(S_{2 h}\right)} f(x) \quad \text { at } \hat{x}=T(x) .
$$

Denote

$$
\hat{S}_{h}=\left\{\hat{x}=T(x): x \in S_{h}\right\}
$$

By direct computation,

$$
\begin{aligned}
& \hat{\nu}\left(\hat{S}_{2 h}\right):=\int_{\hat{S}_{2 h}} d \hat{\nu}=\frac{r^{n}}{\nu\left(S_{2 h}\right)} \frac{\nu\left(S_{2 h}\right)}{r_{1} \cdots r_{n}}=1, \\
& \int_{\hat{S}_{2 h}} \hat{f} d \hat{x}=\frac{r^{n}}{\nu\left(S_{2 h}\right)} \frac{\int_{S_{2 h}} f d x}{r_{1} \cdots r_{n}}=\frac{1}{\nu\left(S_{2 h}\right)} \int_{S_{2 h}} f d x .
\end{aligned}
$$

Let

$$
u^{*}=\nu\left(S_{2 h}\right) \hat{u}=\frac{1}{r}\left[\nu\left(S_{2 h}\right)\right]^{1-\frac{1}{n}}\left[u-\inf _{S_{2 h}} u\right] .
$$

Then we have

$$
L_{\hat{\varphi}}\left[u^{*}\right]=f^{*} \text { in } \hat{S}_{2 h}
$$

where $f^{*}(\hat{x})=r^{n} f(x)$,

$$
\int_{\hat{S}_{2 h}} f^{*} d \hat{x}=1 \text { if } \int_{S_{2 h}} f d x=1
$$

From now on, we let the function $u$ in $(3.19)$ be the Green function $G(\cdot, y)$. We claim that

$$
u^{*} \geq c^{*} \text { on } \partial \hat{S}_{h} .
$$

To prove (3.26), noting that $\hat{S}_{2 h}$ is normalized and $\hat{\varphi}=0$ on $\partial \hat{S}_{2 h}$, by (3.22) and condition $\mathbb{C} \mathbb{G}$ we have

$$
-C_{1} \leq \inf _{\hat{S}_{2 h}} \hat{\varphi} \leq-C_{2},
$$

namely, $\hat{\varphi}$ is normalized up to a constant under control. If (3.26) is not true, by the Harnack inequality, $u^{*}$ is nonnegative but small everywhere on $\partial \hat{S}_{h}$. Recalling that $u^{*} \geq 0$ in $\hat{S}_{2 h}$, by replacing $u^{*}$ by the Green function of $L_{\hat{\varphi}}$ in $\hat{S}_{2 h}$, we may assume that $u^{*}=0$ on $\partial \hat{S}_{2 h}$. Let $w$ be the solution of (3.12). Then $-w>C^{*} u^{*}$ on $\partial \hat{S}_{h}$ for a 
large constant $C^{*}$. Hence $-w>C^{*} u^{*}$ in $\hat{S}_{2 h}-\hat{S}_{h}$. By the divergence structure of $L_{\hat{\varphi}}$ and integrating by parts, we have

$$
\int_{\partial \hat{S}_{2 h}} \hat{a}_{i j} w_{i} \gamma_{j} \geq C^{*} \int_{\partial \hat{S}_{2 h}} \hat{a}_{i j}\left(-u_{i}^{*}\right) \gamma_{j}=C^{*} \int_{\hat{S}_{2 h}} L_{\hat{\varphi}} u^{*}=C^{*},
$$

where $\gamma$ is the unit outer normal and $\left\{\hat{a}_{i j}\right\}$ is the cofactor matrix of $D^{2} \hat{\varphi}$. We reach a contradiction with the second inequality in (3.13).

Denote $S_{k}=S_{\varphi}\left(y, 2^{-k}\right), t_{k}=\inf _{\partial S_{k}} G(\cdot, y), \bar{t}_{k}=\sup _{\partial S_{k}} G(\cdot, y), r_{k}=\left|S_{k}\right|^{1 / n}$. From (3.23) and (3.26), it follows

$$
t_{k+1}-t_{k} \geq c^{*} r_{k}\left[\nu\left(S_{k}\right)\right]^{\frac{1}{n}-1} .
$$

Hence

$$
t_{k+1} \geq c^{*} \sum_{j=1}^{k} r_{j}\left[\nu\left(S_{j}\right)\right]^{\frac{1}{n}-1} .
$$

By the Harnack inequality (3.16),

$$
\bar{t}_{k} \leq C t_{k} \leq C_{1} \sum_{j=1}^{k-1} r_{j}\left[\nu\left(S_{j}\right)\right]^{\frac{1}{n}-1} .
$$

We claim that there exists $m>0$ such that for any $k \geq 0$,

$$
r_{k+m}\left[\nu\left(S_{k+m}\right)\right]^{\frac{1}{n}-1} \geq 2 r_{k}\left[\nu\left(S_{k}\right)\right]^{\frac{1}{n}-1} .
$$

Indeed, by normalizing as in (3.8)-(3.10), it suffices to prove (3.28) for $k=0$. But when $k=0,(3.28)$ follows by the second inequality in assumption (3.14).

By induction and (3.28) it is easy to show that for any $k \geq 1$,

$$
r_{k}\left[\nu\left(S_{k}\right)\right]^{\frac{1}{n}-1} \geq c_{1} \sum_{j=1}^{k-1} r_{j}\left[\nu\left(S_{j}\right)\right]^{\frac{1}{n}-1} .
$$

As a consequence,

$$
t_{k+1} \geq\left(1+\delta_{0}\right) t_{k}
$$

for a positive constant $\delta_{0}$ depending only on $c_{1}$ and $m$.

From (3.29) it follows that

$$
u^{*} \leq c_{1}^{*} \quad \text { on } \partial \hat{S}_{h} .
$$

Indeed, let $\bar{m} \geq 1$ such that $\left(1+\delta_{0}\right)^{\bar{m}}>C$, where $C$ is the constant in the Harnack inequality (3.16). Then we have $\{x \in \Omega: u(x)>t\} \subset S_{\varphi}\left(y, 2^{\bar{m}} h\right)$. Hence $u^{*} \leq 0$ on $\partial \hat{S}_{2^{\bar{m}} h}$. Similar to the proof of (3.26), we obtain (3.30) from Lemma 3.2.

From (3.23), (3.29) and (3.30) it follows that

$$
\frac{1}{r}\left[\nu\left(S_{h}\right)\right]^{1-\frac{1}{n}} t \leq c_{1}^{*} / \delta_{0} .
$$

By the first inequality in assumption (3.14) and noting that $r^{n} \approx\left|S_{2 h}\right| \approx\left|S_{h}\right|$, we have $r \leq\left[\nu\left(S_{h}\right) / C\right]^{1 / n(1+\theta)}$, where the notation $a \approx b$ means $C^{-1} a \leq b \leq C a$ for some constant $C$ under control. Hence

$$
\left[\nu\left(S_{h}\right)\right]^{1-\frac{2+\theta}{n(1+\theta)}} \leq \frac{K}{t}
$$

We have proved (3.15).

By Lemma 3.3 and Lemma 2.1 we have therefore obtain 
THEOREM 3.1. Let $\varphi$ be a smooth, strictly convex function defined in a neighborhood of a bounded domain $\Omega \subset \mathbb{R}^{n}, n \geq 3$. Suppose $\varphi$ satisfies (3.14), and the structure condition $\mathbb{C} \mathbb{G}$. Then for any $u \in C_{0}^{\infty}(\Omega)$,

$$
\left[\int_{\Omega}|u|^{p} d \nu\right]^{1 / p} \leq C\left[\int_{\Omega} a_{i j}(x) u_{i} u_{j} d x\right]^{1 / 2}
$$

where

$$
p=\frac{2 n(1+\theta)}{(n-1)(1+\theta)-1} .
$$

and $C$ depends only on $n$, the constants in (3.14), and the structure condition $\mathbb{C} \mathbb{G}$.

By the first inequality of (3.14) and (3.31) we have

$$
\left|S_{h}\right|^{1+\theta-\frac{2+\theta}{n}} \leq \frac{K}{t}
$$

Hence if $1+\theta-\frac{2+\theta}{n}<1$, namely $\theta<\frac{2}{n-1}$, we obtain

$$
\left|S_{h}\right| \leq K t^{-p / 2}
$$

for $p=\frac{2 n}{(n-1)(1+\theta)-1}>2$. Therefore by Lemma 2.2, we obtain

THEOREM 3.2. Let $\varphi$ be a smooth, strictly convex function defined in in a neighborhood of a bounded domain $\Omega \subset \mathbb{R}^{n}, n \geq 3$. Suppose $\varphi$ satisfies (3.14) with $\theta<\frac{2}{n-1}$. Suppose also that the structure condition $\mathbb{C} \mathbb{G}$ holds. Then for any $u \in C_{0}^{\infty}(\Omega)$,

$$
\left[\int_{\Omega}|u|^{p} d x\right]^{1 / p} \leq C\left[\int_{\Omega} a_{i j}(x) u_{i} u_{j} d x\right]^{1 / 2}
$$

where

$$
p=\frac{2 n}{(n-1)(1+\theta)-1} .
$$

and $C$ depends only on $n$, the constants in (3.14), and the structure condition $\mathbb{C} \mathbb{G}$.

REMARK 3.1. In Theorems 3.1 and 3.2 we don't assume the doubling condition (2.3), as it follows from the condition $\mathbb{C} \mathbb{G}$. Note that if the first inequality of (3.14) holds for all sub-level sets, then necessarily $\theta \geq 0$. Hence $p \leq \frac{2 n}{n-2}$.

REMARK 3.2. In Theorems 3.1 and 3.2, the function $\varphi$ is assumed to be smooth and strictly convex. But the constant $C$ in (3.32) and (3.36) do not depend on the upper and lower bounds of $D^{2} \varphi$. Therefore by approximation, Theorems 3.1 and 3.2 hold for strictly convex functions $\varphi$ provided its cofactor matrix is integrable. More precisely, let $\varphi^{\varepsilon}$ be the mollification of $\varphi$ and let $a_{i j}^{\varepsilon}$ be the cofactor matrix of $D^{2} \varphi^{\varepsilon}$, then $a_{i j}^{\varepsilon}$ converges to $a_{i j}$ weakly as measures [TW2]. In particular, for any continuous function $f_{i j} \in C^{0}(\Omega)$, one has

$$
\int_{\Omega} a_{i j}^{\varepsilon} f_{i j} d x \rightarrow \int_{\Omega} a_{i j} f_{i j} d x
$$

REMARK 3.3. Let $W_{0, \varphi}^{1,2}(\Omega)$ be the completion of $C_{0}^{\infty}(\Omega)$ under the norm

$$
\|u\|_{W_{0, \varphi}^{1,2}(\Omega)}=\left[\int_{\Omega} \sum a_{i j} u_{i} u_{j}\right]^{1 / 2}
$$


Then by the Sobolev inequality (3.32) or (3.36), one can introduce a weak solution to the linearized Monge-Ampere equation (3.1) with $f \in L^{1}(\Omega)$. Note that the matrix $\left\{a_{i j}\right\}$ can be both degenerate and singular. It is inconvenient to introduce a weak solution for (3.1) without a Sobolev type inequality related to the norm (3.39).

Remark 3.4. Here we verify the conditions in Theorems 3.1 and 3.2 for the example (1.5). For the first inequality in (3.14), it suffices to consider the sub-level sets $S_{\varphi}(y, h)$ at $y=0$. We have

$$
\nu=\operatorname{det} D^{2} \varphi \approx\left|x_{1}\right|^{\alpha_{1}} \cdots\left|x_{n}\right|^{\alpha_{n}},
$$

It is easy to compute that

$$
\begin{aligned}
& \left|S_{\varphi}(0, h)\right| \approx h^{\frac{1}{\alpha_{1}+2}+\cdots+\frac{1}{\alpha_{n}+2}}, \\
& \nu\left(S_{\varphi}(0, h)\right) \approx h^{\frac{\alpha_{1}+1}{\alpha_{1}+2}+\cdots+\frac{\alpha_{n}+1}{\alpha_{n}+2}} .
\end{aligned}
$$

Hence $\nu\left(S_{\varphi}(0, h)\right) \approx\left|S_{\varphi}(0, h)\right|^{1+\theta}$ with

$$
\begin{aligned}
1+\theta & =\frac{\frac{\alpha_{1}+1}{\alpha_{1}+2}+\cdots+\frac{\alpha_{n}+1}{\alpha_{n}+2}}{\frac{1}{\alpha_{1}+2}+\cdots+\frac{1}{\alpha_{n}+2}} \\
& =\frac{n}{\frac{1}{\alpha_{1}+2}+\cdots+\frac{1}{\alpha_{n}+2}}-1 .
\end{aligned}
$$

Therefore $\theta<\frac{2}{n-1}$ is equivalent to

$$
\frac{1}{\alpha_{1}+2}+\cdots+\frac{1}{\alpha_{n}+2}>\frac{n-1}{2} .
$$

Similarly, the second inequality of (3.14) holds if

$$
n-1>\frac{1}{\alpha_{1}+2}+\cdots+\frac{1}{\alpha_{n}+2} .
$$

Recall that $\alpha_{i}>-1$ for $i=1,2, \cdots, n$. The function $\nu$ is allowed to be singular.

It is easy to see that (3.41) holds if $\alpha_{i} \leq 0$ for all $i \geq 2$, or if $\alpha_{1} \alpha_{2}<4$ and $\alpha_{i} \leq 0$ for all $i \geq 3$. In particular the Sobolev inequality (3.36) holds for the first example (1.3) for all $\alpha>-1$.

For the second example (1.5), we also note that the exponents $p$ given in (3.33) and (3.37), with $\theta$ given by (3.40) are optimal, as the inequality is invariant under linear transforms which normalizes sub-level sets of $\varphi$. In particular we see that when (3.41) is not satisfied, one cannot expect a Sobolev embedding $W_{0, \varphi}^{1,2}(\Omega) \hookrightarrow L^{p}(\Omega)$ for any $p>2$. It also means the Harnack inequality of Caffarelli and Gutierrez for the linearized Monge-Ampère equation (3.3) cannot be obtained by the De Giorgi or Nash-Moser iterations.

\section{REFERENCES}

[CG1] L.A. Caffarelli and C.E. Gutierrez, Real analysis related to the Monge-Ampére equation, Trans. Amer. Math. Soc., 348 (1996), pp. 1075-1092.

[CG2] L.A. Caffarelli and C.E. Gutierrez, Properties of the solutions of the linearized Monge-Ampere equation, Amer. J. Math., 119 (1997), pp. 423-465.

[C] G. Carron, Inégalités isopérimétriques de Faber-Krahn et conséquences, Actes de la Table Ronde de Géométrie Différentielle, 1992, pp. 205-232. 
[CDG] L. Capogna, D. Danielli, and N. Garofalo, An embedding theorem and the Harnack inequality for nonlinear subelliptic equations, Comm. Partial Diff. Eqns, 18 (1993), pp. 1765-1794.

[CW] S. Chanillo and R.L. Wheeden, Existence and estimates of Green's function for degenerate elliptic equations, Ann. Scuola Norm. Sup. Pisa Cl. Sci.(4), 15 (1988), pp. 309-340.

[FJK] E. Fabes, D. Jerison, And C. Kenig, The Wiener test for degenerate elliptic equations, Ann. Inst. Fourier (Grenoble), 32 (1982), pp. 151-182.

[FS] C. FefFerman And A. SAnchez-CAlle, Fundamental solutions for second order subelliptic operators, Ann. Math., 124 (1986), pp. 247-272.

[FGW1] B. Franchi, S. Gallot, and R.L. Wheeden, Sobolev and isoperimetric inequalities for degenerate metrics, Math. Ann., 300 (1994), pp. 557-571.

[FGW2] B. Franchi, C. Gutierrez, and R. Wheeden, Weighted Sobolev-Poincare inequalities for Grushin type operators, Comm. Partial Diff. Eqns, 19 (1994), pp. 523-604.

[FL] B. Franchi and E. LANCONelli, Hölder regularity theorem for a class of linear nonuniformly elliptic operators with measurable coefficients, Ann. Scuola Norm. Sup. Pisa Cl. Sci., 10 (1983), pp. 523-541.

[GN] N. Garofalo And D.M. Nhieu, Isoperimetric and Sobolev inequalities for Carnot-Carathéodory spaces and the existence of minimal surfaces, Comm. Pure Appl. Math., 49 (1996), pp. 1081-1144.

[GT] D. Gilbarg and N.S. Trudinger, Elliptic partial differential equations of second order, Springer, 1983.

[H] E. Hebey, Nonlinear analysis on manifolds: Sobolev spaces and inequalities, Courant Lecture Notes in Mathematics 5, 1999.

[KM] T. Kilpelainen, And J. Maly, The Wiener test and potential estimates for quasilinear elliptic equations, Acta Math., 172 (1994), pp. 137-161.

[La] D. Labutin, Potential estimates for a class of fully nonlinear elliptic equations, Duke Math. J., 111 (2002), pp. 1-49.

[L1] G.Z. Lu, Weighted Poincaré and Sobolev inequalities for vector fields satisfying Hörmander's condition and applications, Rev. Mat. Iberoamericana, 8 (1992), pp. 367-439.

[L2] G.Z. Lu, Existence and size estimates for the Green's functions of differential operators constructed from degenerate vector fields, Comm. Partial Diff. Eqns, 17 (1992), pp. 12131251.

[LYZ] E. Lutwak, D. Yang, And G.Y. Zhang, Sharp affine $L_{p}$ Sobolev inequalities, J. Diff. Geom., 62 (2002), pp. 17-38.

[MS] J.H. Michael AND L.M. Simon, Sobolev and mean-value inequalities on generalized submanifolds of $R^{n}$, Comm. Pure Appl. Math., 26 (1973), pp. 361-379.

[M] R. Monti, Sobolev inequalities for weighted gradients, Comm. Partial diff. eqns, 31 (2006), pp. 1479-1504.

[P] A.V. Pogorelov, The Minkowski multidimensional problem, J. Wiley, New York, 1978.

[TW1] N.S. Trudinger And X.-J. WAnG, Weak continuity of elliptic operators and its applications in potential theory, Amer. J. Math., 124 (2002), pp. 369-410.

[TW2] N.S. Trudinger And X.-J. Wang, Hessian measures III, J. Funct. Anal., 193 (2002), pp. $1-23$.

[W] X.-J. WAng, Some counterexamples to the regularity of Monge-Ampère equations, Proc. Amer. Math. Soc., 123 (1995), pp. 841-845. 